\title{
Charge-Orbital Ordering and Verwey Transition in Magnetite Measured by Resonant Soft X-Ray Scattering
}

\author{
D. J. Huang, ${ }^{1,2, *}$ H.-J. Lin, ${ }^{1}$ J. Okamoto, ${ }^{1}$ K. S. Chao, ${ }^{2}$ H.-T. Jeng, ${ }^{3}$ G. Y. Guo, ${ }^{4,1}$ C.-H. Hsu, ${ }^{1}$ C.-M. Huang, ${ }^{1}$ D. C. Ling, ${ }^{5}$ \\ W. B. Wu, ${ }^{2}$ C.S. Yang, ${ }^{1}$ and C. T. Chen ${ }^{1,4}$ \\ ${ }^{1}$ National Synchrotron Radiation Research Center, Hsinchu 30076, Taiwan \\ ${ }^{2}$ Department of Electrophysics, National Chiao-Tung University, Hsinchu 30010, Taiwan \\ ${ }^{3}$ Physics Division, National Center for Theoretical Sciences, Hinchu 30013, Taiwan \\ ${ }^{4}$ Department of Physics, National Taiwan University, Taipei 10617, Taiwan \\ ${ }^{5}$ Department of Physics, Tamkang University, Taipei 25137, Taiwan
}

(Received 24 June 2005; published 9 March 2006)

\begin{abstract}
We report experimental evidence for the charge-orbital ordering in magnetite below the Verwey transition temperature $T_{\mathrm{V}}$. Measurements of $\mathrm{O} K$-edge resonant $\mathrm{x}$-ray scattering on magnetite reveal that the $\mathrm{O} 2 p$ states in the vicinity of the Fermi level exhibit a charge-orbital ordering along the $c$ axis with a spatial periodicity of the doubled lattice parameter of the undistorted cubic phase. Such a charge-orbital ordering vanishes abruptly above $T_{\mathrm{V}}$ and exhibits a thermal hysteresis, correlating closely with the Verwey transition in magnetite.
\end{abstract}

DOI: 10.1103/PhysRevLett.96.096401

PACS numbers: 71.30.+h, 61.10.-i, 71.28.+d

Elucidating the nature of phase transitions is one of the most important subjects in condensed-matter physics. Many phase transitions exhibited by electron-correlated materials are closely related to charge and orbital orderings $[1,2]$, which manifest themselves in the spatial localization of the charge carriers on certain sites and in the periodic arrangement of specific electron orbitals, respectively. One important example which has fascinated generations of solid-state physicists is the metal-to-insulator transition in magnetite $\left(\mathrm{Fe}_{3} \mathrm{O}_{4}\right)$, known as the Verwey transition [3]. Such a transition was first interpreted as a charge order-disorder transition in which the valence electrons order themselves over the octahedral sites (B sites) to form a $\mathrm{Fe}^{2+}$ and $\mathrm{Fe}^{3+}$ superstructure in the insulating phase [4]. Despite intensive investigations over half a century, the existence of charge ordering in magnetite remains controversial [5-9]. The mechanism of the Verwey transition is a fundamental yet unresolved problem.

Above the transition temperature $T_{\mathrm{V}}, \mathrm{Fe}_{3} \mathrm{O}_{4}$ crystallizes in an inverse spinel structure with a cubic lattice. Upon cooling through $T_{\mathrm{V}}, \mathrm{Fe}_{3} \mathrm{O}_{4}$ undergoes the Verwey transition in which the electrical conductivity decreases by 2 orders of magnitude and the lattice distorts from cubic to monoclinic structure. Manifestations of the Verwey transition also include abrupt changes in magnetic susceptibility and specific heat across the critical temperature. The low-temperature phase of magnetite has been regarded as a classic example of the charge ordering in transition-metal oxides. On the other hand, electron-phonon interactions [10-12] have also been attributed to be the driving force of the Verwey transition, as the freezing of phonon modes relieves an instability of the Fermi surface nesting via a mechanism involving charge-density waves below $T_{\mathrm{V}}$, and therefore increases the resistivity.
The mechanism of the Verwey transition is intricate, because neither charge-orbital ordering of valence electrons nor the soft phonon mode has been observed. Many experiments cast strong doubt on the existence of charge ordering in magnetite. In contrast, refinements of x-ray and neutron diffraction $[13,14]$ suggest the existence of a small charge disproportionation with a modulation vector $\mathbf{q}=$ $(001)_{c}$ and $\left(00 \frac{1}{2}\right)_{c}$ in units of $2 \pi / a$ with $a$ denoting the lattice parameter of the undistorted cubic phase (script " $c$ " denotes the high-temperature cubic structure throughout). Such suggestion is supported by LDA + U band-structure calculations [15] which also predict that $\mathrm{Fe}_{3} \mathrm{O}_{4}$ has a charge-orbital ordered ground state [16,17].

The detection of temperature-dependent electronic states across $T_{\mathrm{V}}$ in magnetite is thus a decisive measurement for revealing the mechanism of the Verwey transition. In this Letter, we show ordering of the $\mathrm{O} 2 p$ states with a modulation vector $\left(00 \frac{1}{2}\right)_{c}$ at temperature below $T_{\mathrm{V}}$. Because of the hybridization between the $\mathrm{O} 2 p$ and the $\mathrm{Fe} 3 d$, our finding provides experimental evidence for the existence of charge-orbital ordering in magnetite.

We performed resonant soft $\mathrm{x}$-ray scattering on single crystals of $\mathrm{Fe}_{3} \mathrm{O}_{4}$ with the ultrahigh vacuum soft $\mathrm{x}$-ray diffractometer and the elliptically polarized undulator beam line of National Synchrotron Radiation Research Center in Taiwan. The crystals were fully characterized with $\mathrm{x}$-ray diffraction and resistivity measurements which show a sharp Verwey transition. $\mathrm{Fe}_{3} \mathrm{O}_{4}(001)$ crystals were polished with fine $\mathrm{Al}_{2} \mathrm{O}_{3}$ powder to achieve a mirrorlike surface.

Soft x-ray scattering has been shown to be effective for probing charge and orbital ordering of transition-metal oxides [18-21]. In a resonant elastic x-ray scattering, a core electron is virtually excited to some intermediate 
states above the Fermi level, and subsequently decays to the same core level. The intensity $I(\mathbf{q}, \hbar \omega)$ of resonant elastic x-ray scattering with energy $\hbar \omega$ and momentum transfer $\mathbf{q}$ is determined by the structure form factor and the resonant atomic scattering tensor [22,23], where $\mathbf{q}$ is defined as $\mathbf{k}^{\prime}-\mathbf{k}$ with $\mathbf{k}^{\prime}$ and $\mathbf{k}$ denoting the wave vectors of the scattered and the incoming soft $\mathrm{x}$ rays, respectively. As for the detection of charge ordering in $\mathrm{Fe}_{3} \mathrm{O}_{4}$, one can employ $\mathrm{O} K$-edge resonant elastic $\mathrm{x}$-ray scattering to examine whether the $\mathrm{O} 2 p$ states exhibit a spatially periodic ordering, because the $\mathrm{O} 2 p$ states hybridize with the Fe $3 d$ states which are suggested to have a charge ordering with a propagation vector $\left(00 \frac{1}{2}\right)_{c}$ [13]. In addition, the structure factor of scattering with $\mathbf{q}=\left(00 \frac{1}{2}\right)_{c}$ is rather insensitive to the resonance arising from the oxygen atoms in $\mathrm{Fe}_{3} \mathrm{O}_{4}$ [24]; hence the resonant $\mathrm{O} K$-edge scattering with $\mathbf{q}=\left(00 \frac{1}{2}\right)_{c}$ can be used to separate the contribution of charge-orbital ordering from the effect of the pure lattice distortion.

Figure 1 displays the photon-energy-dependent resonant scattering intensities of $\mathrm{Fe}_{3} \mathrm{O}_{4}$ around the $\mathrm{O} K$ edge with constant momentum transfer. The data were measured at sample temperature of $80 \mathrm{~K}$ with the $E$ vector in the scattering plane and its surface projection along the $a$ axis. Remarkably, the $\left(00 \frac{1}{2}\right)_{c}$ scattering at energy below the absorption edge (in a narrow energy window from 528.5 to $530.5 \mathrm{eV}$ ) enhances dramatically, whereas the scattering intensity is insignificant throughout the absorption white lines (from 530.5 to $536 \mathrm{eV}$ ). The observed $\left(00 \frac{1}{2}\right)_{c}$ resonance scattering is a manifestation of $\mathrm{O} 2 p$ ordering instead of the doubling of the unit cell. If the resonant scattering was dominated by a structural effect, the $\left(00 \frac{1}{2}\right)_{c}$ scattering intensity would be observable both

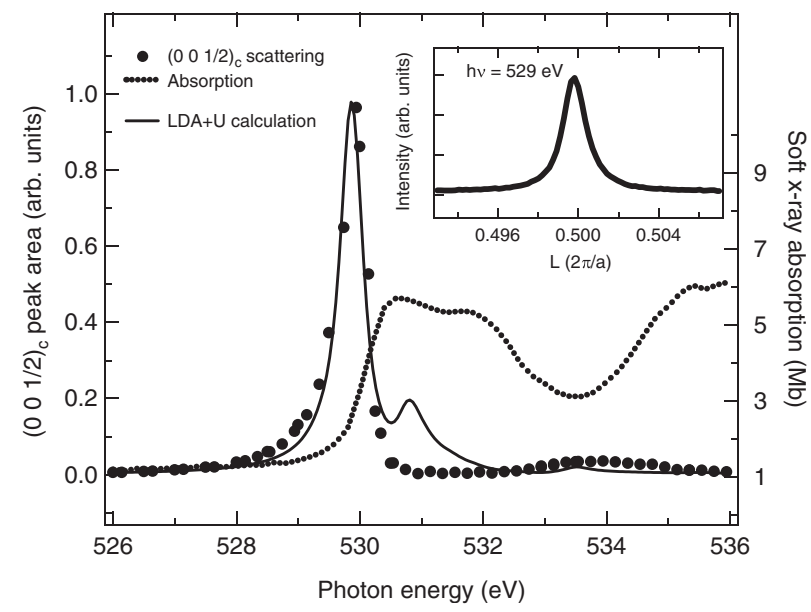

FIG. 1. Photon-energy dependence of the $\left(00 \frac{1}{2}\right)_{c}$ scattering intensity (circles) and soft x-ray absorption cross section (dotted line) of $\mathrm{Fe}_{3} \mathrm{O}_{4}$ around the $\mathrm{O} K$ edge $(1 s \rightarrow 2 p)$. The inset displays the $q_{z}$ scan at photon energy of $529 \mathrm{eV}$. The scattering measurements are compared with calculated resonant spectrum (solid line) obtained by employing the results of LDA $+U$ calculations as discussed in the text. below the $\mathrm{O} K$-edge absorption edge and throughout the absorption white line. In contrast, the ordering of the $\mathrm{O} 2 p$ orbitals with a periodicity of $2 a$ will lead to the enhancements of $I(\mathbf{q}, \hbar \omega)$ only at photon energy $\hbar \omega$ corresponding to the excitations of these ordered orbitals and at $\mathbf{q}=$ $\left(00 \frac{1}{2}\right)_{c}$. Our measurements therefore provide evidence for the existence of the superstructure of the ordered $\mathrm{O} 2 p$ orbital states in the vicinity of the Fermi level.

To ensure that the measurements of resonant scattering are not obscured by the self-absorption effect of soft x-ray in the sample, we compared the correlation length $\xi$ of the $\mathrm{O} 2 p$ ordering and the penetration depth of soft $\mathrm{x}$ ray. Here we define the correlation length as $\xi=\frac{a}{2 \pi} \frac{1}{\Delta q}$ with $\Delta q$ denoting the full width at half maximum of the $q_{z}$ scan in reciprocal lattice units of $2 \pi / a$. The inset of Fig. 1 depicts the width of the diffraction peak in the $q_{z}$ scan at the onset energy of the resonant scattering, giving rise to a correlation length $\xi \sim 900 \AA$. Deduced from the measured absorption cross section, the penetration depths of photons with the energy between 528 and $531 \mathrm{eV}$ are greater than $1300 \AA$, which is larger than the measured correlation length. In the following discussions on the polarization and temperature dependence of $\mathrm{O} 2 p$ ordering, we present data measured with photon energy of $529 \mathrm{eV}$, at which the penetration depth is $5500 \AA$, to avoid the experimental complication caused by the sample self-absorption effect.

The observed resonance can be an effect of either pure charge modulation or charge-orbital ordering. The latter would lead to a resonant scattering depending on the polarization of the incident soft $\mathrm{x}$ ray. To disentangle this uncertainty, we calculated spatial distribution of $\mathrm{O} 2 p$ orbitals using LDA $+\mathrm{U}$ calculations on $\mathrm{Fe}_{3} \mathrm{O}_{4}$. Our calculations [25] show that distorted $\mathrm{Fe}_{3} \mathrm{O}_{4}$ exhibits localized O $2 p$ orbitals in the vicinity of the Fermi level with a modulation vector $\left(00 \frac{1}{2}\right)_{c}$, i.e., O $2 p$ charge-orbital ordering, in addition to the charge-orbital ordering of the Fe $3 d$ predicted previously $[16,17]$. Figure 2 illustrates the monoclinic crystal structure of $\mathrm{Fe}_{3} \mathrm{O}_{4}$ with $P 2 / c$ symmetry and the three-dimensional isosurfaces of unoccupied density of states of the $\mathrm{O} 2 p$ within corner-sharing $\mathrm{Fe}-\mathrm{O}$ cubes of octahedral iron and oxygen. The distributions of unoccupied $\mathrm{O} 2 p$ orbitals are calculated within spheres of the oxygen sites with a radius of $0.9 \AA$. With the LDA + U calculations, we also calculated the resonant x-ray scattering spectrum at the $\mathrm{O} K$ edge of $\mathrm{Fe}_{3} \mathrm{O}_{4}[22,23,26]$, as plotted in the solid curve of Fig. 1. The calculations qualitatively explain the resonance feature of the measured spectrum, further corroborating that the observed resonance arises from the $\mathrm{O} 2 p$ charge-orbital ordering.

In addition, we found that the $\left(00 \frac{1}{2}\right)_{c}$ resonant scattering at the $\mathrm{O} K$ edge exhibits a polarization dependence. Figure 3 shows that the $\left(00 \frac{1}{2}\right)_{c}$ scattering spectra taken with the $\mathbf{E}$ vector of the incident soft $\mathrm{x}$ ray in the scattering plane $\left(\mathbf{E}_{\pi}\right)$ and perpendicular to the scattering plane $\left(\mathbf{E}_{\sigma}\right)$. As the polarization of the soft $\mathrm{x}$ ray is rotated from $\mathbf{E}_{\pi}$ to $\mathbf{E}_{\sigma}$, the scattering intensity is reduced. Such a polarization 


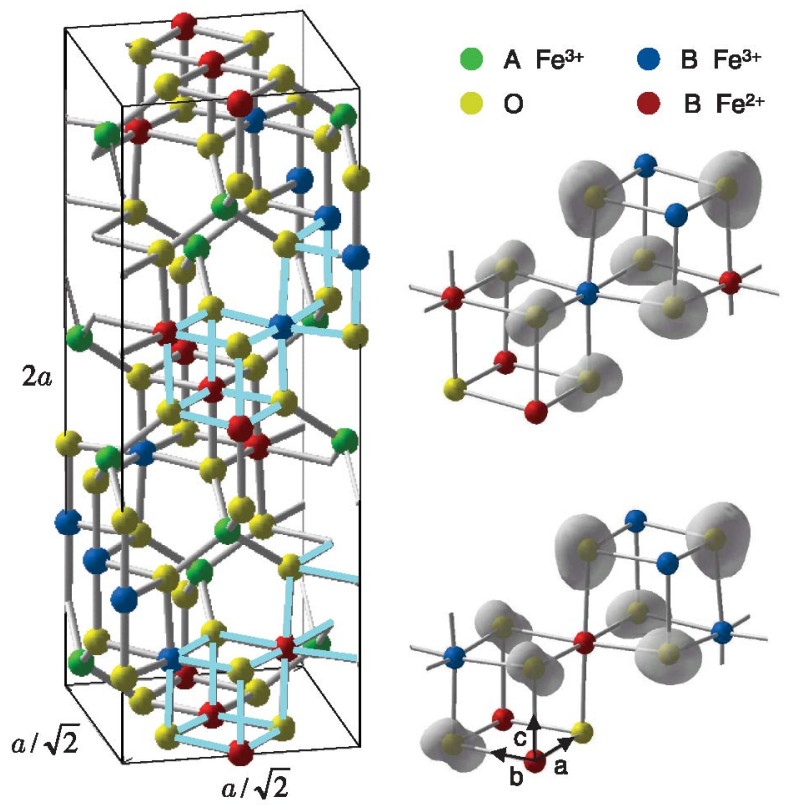

FIG. 2 (color). Left: monoclinic crystal structure of $\mathrm{Fe}_{3} \mathrm{O}_{4}$ in the low-temperature phase corresponding to a subcell of $\frac{a}{\sqrt{2}} \times$ $\frac{a}{\sqrt{2}} \times 2 a$ with $P 2 / c$ symmetry. Right: $3 \mathrm{D}$ isosurfaces of unoccupied density of states of the $\mathrm{O} 2 p$ integrated between the Fermi level and $1 \mathrm{eV}$ above within the corresponding $\mathrm{Fe}_{3} \mathrm{O}_{4}$ cubes highlighted in the crystal structure. For simplicity, we denote the octahedral Fe site with nominal $2+$ and $3+$ valences as $\mathrm{Fe}^{2+}$ and $\mathrm{Fe}^{3+}$, respectively.

dependence can be explained by LDA $+\mathrm{U}$ calculations. $\mathrm{O}$ $2 p$ orbitals at elevations of $z=0$ and $z=a\left(z=\frac{a}{4}\right.$ and $z=$ $\frac{5 a}{4}$ ) are nearly orthogonal (Fig. 2); the projection of the $\mathrm{O}$ $2 p$ orbitals along the direction of $\mathbf{E}_{\pi}$ has a stronger contrast between these elevations than that of the $\mathrm{O} 2 p$ orbitals along the direction of $\mathbf{E}_{\sigma}$. The results of LDA $+\mathrm{U}$ calcu-

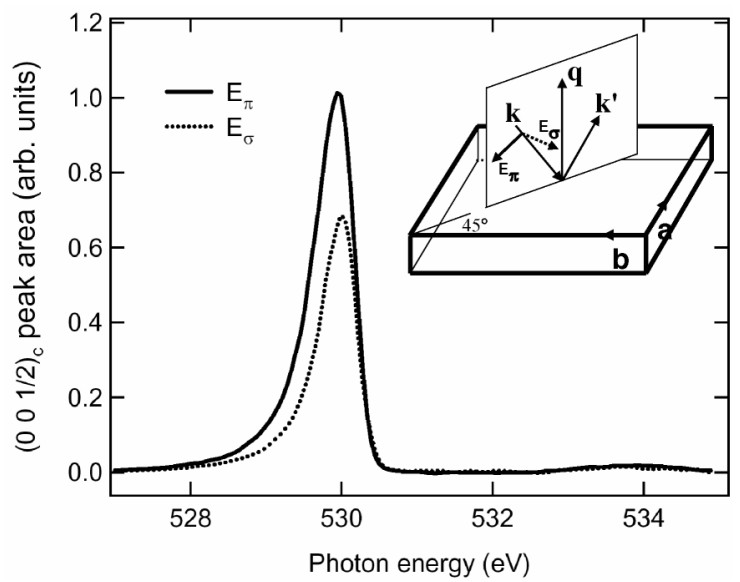

FIG. 3. Polarization dependence of the $\left(00 \frac{1}{2}\right)_{c} \mathrm{O} K$-edge scattering intensity. The data were measured at sample temperature of $100 \mathrm{~K}$ by using photons with polarization in the scattering plane $\left(\mathbf{E}_{\pi}\right)$ and perpendicular to the scattering plane $\left(\mathbf{E}_{\sigma}\right)$ defined by the incoming wave vector $\mathbf{k}$ and the scattered wave vector $\mathbf{k}^{\prime}$, as illustrated in the inset. lations are thus consistent with our polarization-dependent measurement, lending further support for the existence of superstructure of the $\mathrm{O} 2 p$ states in magnetite. Because of the $p$ - $d$ hybridization, the superstructure of the $\mathrm{O} 2 p$ states is a manifestation of the orbital ordering of Fe $3 d$ states. Our measurements thus provide experimental evidence for the existence of charge-orbital ordering in magnetite below the Verwey transition temperature $T_{\mathrm{V}}$.

After experimentally establishing the existence of charge-orbital ordering, we scrutinized the nature of the Verwey transition by carrying out temperature-dependent measurements. The $\left(00 \frac{1}{2}\right)_{c}$ superstructure peak also exhibits a temperature hysteresis that is the characteristic of a first-order transition, similar to the hysteresis of resistivity. Figure 4 displays resistivity and the intensities of the $\left(00 \frac{1}{2}\right)_{c} \mathrm{O} K$-edge resonant scattering of $\mathrm{Fe}_{3} \mathrm{O}_{4}$ measured simultaneously for temperatures across the Verwey temperature $116.5 \mathrm{~K}$. Upon increasing the temperature and concurrently with the decrease of resistivity, the scattering intensity decreases abruptly at $T_{\mathrm{V}}$ and diminishes completely at temperature beyond $119 \mathrm{~K}$, manifesting the disappearance of charge-orbital ordering at temperature slightly higher than $T_{\mathrm{V}}$. The $\mathrm{O} 2 p$ states at the Fermi level contribute to the electric conduction above $T_{\mathrm{V}}$ and undergo a dramatic change upon cooling below the Verwey temperature at which these states form an orbital ordering, opening up an energy gap $[16,17,27,28]$ and thus leading to the insulating phase. Such charge-orbital ordering is fur-
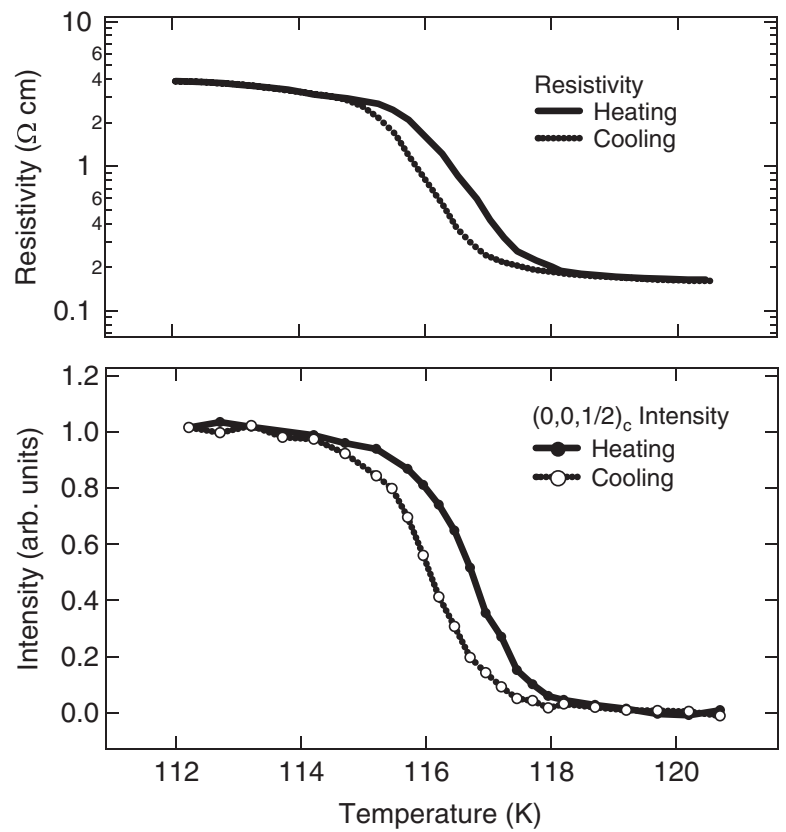

FIG. 4. Temperature-dependent resistivity (upper panel) and the $\left(00 \frac{1}{2}\right)_{c} \mathrm{O} K$-edge scattering intensity (lower panel) of $\mathrm{Fe}_{3} \mathrm{O}_{4}$ across the Verwey transition. The $\left(00 \frac{1}{2}\right)_{c}$ measurements in heating and the cooling are plotted with connecting solid and dotted lines for visual guidance, respectively. The scattering data were measured with photon energy of $529 \mathrm{eV}$ and with the $\mathbf{E}$ vector in the scattering plane and its projection along the a axis. 
ther stabilized by the lattice distortion. With the results of soft $\mathrm{x}$-ray resonant scattering and $\mathrm{LDA}+\mathrm{U}$ calculations, we found that the ground state of $\mathrm{Fe}_{3} \mathrm{O}_{4}$ at $T=0 \mathrm{~K}$ is a charge-orbital ordered state and this ordering is melted by the thermal fluctuation of lattice when the temperature increases above $T_{\mathrm{V}}$. Our results unravel that the $\left(00 \frac{1}{2}\right)_{c}$ scattering has the same temperature dependence as that of resistivity, suggesting that the Verwey transition is a transition of charge-orbital ordering.

To conclude, we found experimental evidence for the charge-orbital ordering in magnetite. $\mathrm{O} K$-edge resonant $\mathrm{x}$-ray scattering reveals that the $\mathrm{O} 2 p$ orbitals in the vicinity of the Fermi level exhibit an ordering with a spatial periodicity of the doubled lattice parameter of the undistorted cubic phase. Our temperature-dependent measurements unravel the electronic nature of the Verwey transition and demonstrate that charge-orbital ordering plays an important role in the Verwey transition of $\mathrm{Fe}_{3} \mathrm{O}_{4}$, suggesting that the Verwey transition is a transition of charge-orbital ordering.

We thank C.H. Chen, A. Fujimori, H.H. Hung, K.S. Liang, C. Y. Mou, and L. H. Tjeng for valuable discussions, and staffs of NSRRC, particularly, H. W. Fu, C. S. Chung, and L. Lee for excellent technical support. We also acknowledge C. Schuessler-Langeheine, J. Schlappa, and C.F. Chang from the University of Cologne for their skillful technical assistance with the scattering measurements. This work was supported in part by the National Science Council of Taiwan.

*Email address: djhuang@ nsrrc.org.tw

[1] M. Imada, A. Fujimori, and Y. Tokura, Rev. Mod. Phys. 70, 1039 (1998).

[2] Y. Tokura and N. Nagaosa, Science 288, 462 (2000).

[3] For review on the research of the Verwey transition, see F. Walz, J. Phys. Condens. Matter 14, R285 (2002); J. Garcia and G. Subias, J. Phys. Condens. Matter 16, R145 (2004).

[4] E. J. W. Verwey and P. W. Haayman, Physica (Amsterdam) 8, 979 (1941).

[5] K. Siratori, Y. Shii, Y. Morii, S. Funahashi, S. Todo, and A. Yanase, J. Phys. Soc. Jpn. 67, 2818 (1998).

[6] P. Novak, H. Stepankova, J. Englich, J. Kohout, and V. A. M. Brabers, Phys. Rev. B 61, 1256 (2000).

[7] J. Garcia, G. Subias, M. G. Proietti, H. Renevier, Y. Joly, J. L. Hodeau, J. Blasco, M. C. Sanchez, and J. F. Berar, Phys. Rev. Lett. 85, 578 (2000).
[8] J. Garcia, G. Subias, M. G. Proietti, J. Blasco, H. Renevier, J. L. Hodeau, and Y. Joly, Phys. Rev. B 63, 054110 (2001).

[9] G. Subias, J. Garcia, J. Blasco, M. Grazia Proietti, H. Renevier, and M.C. Sanchez, Phys. Rev. Lett. 93, 156408 (2004).

[10] F. Mott, Philos. Mag. B 42, 327 (1980).

[11] Y. Yamada, Philos. Mag. B 42, 377 (1980).

[12] A. Yanase and N. Hamada, J. Phys. Soc. Jpn. 68, 1607 (1999).

[13] J. P. Wright, J. P. Attfield, and P. G. Radaelli, Phys. Rev. Lett. 87, 266401 (2001).

[14] J. P. Wright, J. P. Attfield, and P. G. Radaelli, Phys. Rev. B 66, 214422 (2002).

[15] LDA + U stands for the local density approximation explicitly including the on-site Coulomb interactions.

[16] I. Leonov, A. N. Yaresko, V. N. Antonov, M. A. Korotin, and V. I. Anisimov, Phys. Rev. Lett. 93, 146404 (2004).

[17] H. T. Jeng, G. Y. Guo, and D. J. Huang, Phys. Rev. Lett. 93, 156403 (2004).

[18] P. Abbamonte, L. Venema, A. Rusydi, G. A. Sawatzky, G. Logvenov, and I. Bozovic, Science 297, 581 (2002).

[19] S. B. Wilkins, P. D. Spencer, P. D. Hatton, S. P. Collins, M. D. Roper, D. Prabhakaran, and A. T. Boothroyd, Phys. Rev. Lett. 91, 167205 (2003).

[20] K. J. Thomas, J. P. Hill, S. Grenier, Y.-J. Kim, P. Abbamonte, L. Venema, A. Rusydi, Y. Tomioka, Y. Tokura, D.F. McMorrow, G. Sawatzky, and M. van Veenendaal, Phys. Rev. Lett. 92, 237204 (2004).

[21] P. Abbamonte, G. Blumberg, A. Rusydi, A. Gozar, P. G. Evans, T. Siegrist, L. Venema, H. Eisaki, E. D. Isaacs, and G. A. Sawatzky, Nature (London) 431, 1078 (2004).

[22] M. Takahashi, M. Usuda, and J. Igarashi, Phys. Rev. B 67, 064425 (2003).

[23] N. Binggeli and M. Altarelli, Phys. Rev. B 70, 085117 (2004).

[24] The difference in the intensity due to the change of structure factor between photons of energy below and above the $\mathrm{O} K$ edge is negligible, i.e., (1$\left.\frac{\left|\sum_{j}\left(f_{\mathrm{Fe}} e^{i \mathbf{q} \cdot \mathbf{r}_{j}}+f_{\mathrm{O}} e^{i \mathbf{q} \cdot \mathbf{r}_{j}}\right)\right|^{2}}{\left|\sum_{j}\left(f_{\mathrm{Fe}} e^{i \mathbf{q} \cdot \mathbf{r}_{j}}+R \cdot f_{\mathrm{O}} e^{i \mathbf{q} \cdot \mathbf{r}_{j}}\right)\right|^{2}}\right) \ll 1$, where $f_{\mathrm{Fe}}$ and $f_{\mathrm{O}}$ are, respectively, the atomic form factors of $\mathrm{Fe}$ and $\mathrm{O}$, and $R$ is the factor accounting for the enhancement of the form factor of oxygen. In addition, $\vec{q}=\left(00 \frac{1}{2}\right)_{c}$ and the summation runs over all sites of $\mathrm{Fe}$ and $\mathrm{O}$ in $\mathrm{Fe}_{3} \mathrm{O}_{4}$.

[25] See Ref. [17] for the calculation methods; detailed results will be published elsewhere (H.-J. Jeng et al.)

[26] G. Y. Guo et al. (to be published).

[27] A. Chainani, T. Yokoya, T. Morimoto, T. Takahashi, and S. Todo, Phys. Rev. B 51, 17976 (1995).

[28] S. K. Park, T. Ishikawa, and Y. Tokura, Phys. Rev. B 58, 3717 (1998). 